\title{
Energy Content of Gravitation as a Way to Quantify Both Entropy and Information Generation in the Early Universe
}

\author{
Andrew Walcott Beckwith \\ Department of Physics, Chongqing University, Chongqing, China \\ E-mail: beckwith@iibep.org,abeckwith@uh.edu \\ Received January 10, 2011; revised February 21, 2011; accepted February 23, 2011
}

\begin{abstract}
Based upon Alcubierre's formalism about energy flux of gravitational waves, as well as Saunder's treatment of temperature dependence of the Hubble parameter in the early universe, we initiate a particle count treatment of gravitons, and subsequent entropy generation which gives, via the standard model treatment of the FRW metric a way to explain/justify a value of entropy of the order of $S \sim 10^{6}-10^{7}$ at the very onset of inflation.
\end{abstract}

Keywords: Graviton, Hubble Parameter, Entropy Generation

\section{Introduction}

The supposition advanced in this article is that relic energy flux initially is central to making predictions as to $S_{\text {entropy }} \sim n_{f}$, where $n_{f}$ is a "particle count" per phase space 'volume' in the beginning of inflation. The author is aware of how many researchers have linked relic GW as to initial phase transitions as to the electro weak phase transition, in cosmology. The supposition is that $S \sim 10^{6}-10^{7}$ is demonstrable as an initial entropy/information count in the onset of inflation if the Weyl scalar is initially time independent.

\subsection{What Can be Said about Gravitational Wave Density Value Detection?}

We begin with a use of particle count $n_{f}$ for a way to present initial GW relic inflation density using the definition given by Maggiore [1] as a way to state that a particle count algorithm is de rigor experimentally. And that the first place to start would be in obtaining a way to quantify $n_{f}$ generation in relic conditions, as a way of showing a linkage between relic GW generation, entropy, and energy flux values for the onset of inflation. We begin with

$$
\Omega_{g w} \equiv \frac{\rho_{g w}}{\rho_{c}} \equiv
$$

$$
\int_{f=0}^{f=\infty} d(\log f) \cdot \Omega_{g w}(f) \Rightarrow h_{0}^{2} \Omega_{g w}(f) \cong 3.6 \cdot\left[\frac{n_{f}}{10^{37}}\right] \cdot\left(\frac{f}{1 k H z}\right)^{4}
$$

where $n_{f}$ is the frequency-based numerical count of gravitons per unit phase space. While Maggiore's explanation [1], and his treatment of gravitational wave density is very good, the problem we have is that any relic conditions for GW involve stochastic back ground, and also that many theorists have fixated upon either turbulence/and or other forms of plasma induced generation of shock waves, as stated by Duerrer [2] and others looking at the electro weak transition as a GW generator. The energy flux formalism of Alcubierre [3] is a natural way to obtain a criteria which we think explains how ultra low values of entropy, as given by Smoot [4], and others in the Ercole Chalonge Paris colloquia 2007, would arise in initial inflationary cosmology.

In doing so, we will expand upon a counting algorithm for entropy as given by both the author and Y. J. Ng [5], which will, when combined with an expression of energy carried per graviton complete our analysis of the relative importance of particle counting, GW energy, energy per graviton and the linkage of all these factors to initially low entropy.

The author suggests that $n_{f}$ may also depend upon the interaction of gravitons with neutrinos in plasma during early-universe nucleation, as modeled by M. Marklund et 
al [6]. But the main datum to consider would be in analyzing an expression given by Alcubierre's [3] formalism about energy flux, assuming that there is a solid angle for energy distribution $\Omega$ for the energy flux to travel through.

$$
\frac{d E}{d t}=[\lim r \rightarrow \infty]\left[\frac{r^{2}}{16 \pi}\right] \oint\left|\int_{-\infty}^{t} \Psi_{4} d t^{\prime}\right|^{2} \cdot d \Omega
$$

The expression $\Psi_{4}$ is a Weyl scalar which we will write in the form of. (See Equation (3)).

Our assumptions are simple, that if the energy flux expression is to be evaluated properly, before the electro weak phase transition, that time dependence of both $h^{+}$and $h^{x}$ is miniscule and that initially $h^{+} \approx h^{x}$, so as to initiate a re write of Equation (4) below as

$$
\Psi_{4} \cong-\frac{1}{4} \cdot\left[+\partial_{r}^{2} h^{+}\right] \cdot(-1+i)
$$

The upshot, is that the initial energy flux about the inflationary regime would lead to looking at

$$
\left|\int_{-\infty}^{t} \Psi_{4} d t^{\prime}\right| \approx\left|\frac{1}{2} \cdot\left[+\partial_{r}^{2} h^{+}\right]\right| \cdot\left(\tilde{n} \cdot t_{\text {Planck }}\right)
$$

This will lead to an initial energy flux at the onset of inflation which will be presented as

$$
\frac{d E}{d t}=\left[\frac{r^{2}}{64 \pi}\right] \cdot\left|+\partial_{r}^{2} h^{+}\right|^{2} \cdot\left[\tilde{n} \cdot t_{\text {Planck }}\right]^{2} \cdot \Omega
$$

If we are talking about an initial energy flux, we then can approximate the above as

$$
E_{\text {initial-flux }} \cong\left[\frac{r^{2}}{64 \pi}\right] \cdot\left|+\partial_{r}^{2} h^{+}\right|^{2} \cdot\left[\tilde{n} \cdot t_{\text {Planck }}\right]^{3} \cdot \Omega_{\text {effective }}
$$

Inputs into both the expression $\left|\partial_{r}^{2} h^{+}\right|$, as well as $\Omega_{\text {effective }}$ will comprise the rest of this document, plus our conclusions. The derived value of $\Omega_{\text {effective }}$ as well as $E_{\text {initial-flux }}$ will be tied into a way to present energy per graviton, as a way of obtaining $n_{f}$ The $n_{f}$ value so obtained, will be used to make a relationship, using Y. J. Ng's entropy [5] counting algorithm of roughly $S_{\text {entropy }} \sim n_{f}$. We assert that in order to obtain $S_{\text {entropy }} \sim n_{f}$ from initial graviton production, as a way to quantify $n_{f}$, that a small mass of the graviton can be assumed.

\section{Does the Graviton Have Small Mass Initially? Seeming Violation of the Correspondence Principle and a Macro Effect from Small Graviton Mass}

We begin our inquiry by initially looking at a modification of what was presented by R. Maartens $[7,8]$

$$
m_{n}(\text { Graviton })=\frac{n}{L}+10^{-65} \text { grams }
$$

Note that Rubakov [9] writes KK graviton representation as, after using the following normalization $\int \frac{d z}{a(z)} \cdot\left[h_{m}(z) \cdot h_{\tilde{m}}(z)\right] \equiv \delta(m-\tilde{m})$ where $J_{1}, J_{2}, N_{1}, N_{2}$ are different forms of Bessel functions, to obtain the KK graviton/DM candidate representation along RS dS brane world. (Equation (9)).

This Equation (9) is for $\mathrm{KK}$ gravitons having a $\mathrm{TeV}$ magnitude mass $M_{Z} \sim k$ (i.e. for mass values at .5 $\mathrm{TeV}$ to above a $\mathrm{TeV}$ in value) on a negative tension $\mathrm{RS}$ brane. What would be useful would be managing to relate this $\mathrm{KK}$ graviton, which is moving with a speed proportional to $H^{-1}$ with regards to the negative tension brane with $h \equiv h_{m}(z \rightarrow 0)=$ const $\cdot \sqrt{\frac{m}{k}}$ as an initial starting value for the KK graviton mass, before the KK graviton, as a 'massive' graviton moves with velocity $H^{-1}$ along the RS dS brane. If so, and if $h \equiv h_{m}(z \rightarrow 0)=$ const $\cdot \sqrt{\frac{m}{k}}$ represents an initial state, then one may relate the mass of the KK graviton, moving at high speed, with the initial rest mass of the graviton, which in four space in a rest mass configuration would have a mass lower in value, i.e. of $m_{\text {graviton }}(4-\operatorname{Dim} G R) \sim$ $10^{-48} \mathrm{eV}$, as opposed to $M_{X} \sim M_{K K-\text { Graviton }} \sim .5 \times 10^{9} \mathrm{eV}$. Whatever the range of the graviton mass, it may be a way to make sense of what was presented by Dubovsky et al. [10] who argue for graviton mass using CMBR measurements, of $M_{K K \text {-Graviton }} \sim 10^{-20} \mathrm{eV}$ Dubosky et al. [10] results can be conflated with Alves et al. [9] arguing that non zero graviton mass may lead to an acceleration of our present universe, in a manner usually conflated with

$$
\begin{gathered}
\Psi_{4}=-\frac{1}{4} \cdot\left[\partial_{t}^{2} h^{+}-2 \partial_{t} \partial_{r} h^{+}+\partial_{r}^{2} h^{+}\right]+\frac{i}{4} \cdot\left[\partial_{t}^{2} h^{x}-2 \partial_{t} \partial_{r} h^{x}+\partial_{r}^{2} h^{x}\right] \\
h_{m}(z)=\sqrt{m / k} \cdot \frac{J_{1}(m / k) \cdot N_{2}([m / k] \cdot \exp (k \cdot z))-N_{1}(m / k) \cdot J_{2}([m / k] \cdot \exp (k \cdot z))}{\sqrt{\left[J_{1}(m / k)\right]^{2}+\left[N_{1}(m / k)\right]^{2}}}
\end{gathered}
$$


$\mathrm{DE}$, i.e. their graviton mass would be about

$m_{\text {graviton }}(4-\operatorname{Dim} G R) \sim 10^{-48} \times 10^{-5} \mathrm{eV} \sim 10^{65}$

grams.

Also Equation (10) will be the starting point used for a KK tower version of Equation (11) below. So from Maarten's [8] 2005 paper,

$$
\dot{a}^{2}=\left[\left(\frac{\tilde{\kappa}^{2}}{3}\left[\rho+\frac{\rho^{2}}{2 \lambda}\right]\right) a^{2}+\frac{\Lambda \cdot a^{2}}{3}+\frac{m}{a^{2}}-K\right]
$$

Maartens [8] also gives a $2^{\text {nd }}$ Friedman equation, as

$$
\dot{H}^{2}=\left[-\left(\frac{\tilde{\kappa}^{2}}{2} \cdot[p+\rho] \cdot\left[1+\frac{\rho^{2}}{\lambda}\right]\right)+\frac{\Lambda \cdot a^{2}}{3}-2 \frac{m}{a^{4}}+\frac{K}{a^{2}}\right]
$$

Also, if we are in the regime for which $\rho \cong-P$, for red shift values $\mathrm{z}$ between zero to 1.0-1.5 with exact equality, $\rho=-P$, for $\mathrm{z}$ between zero to .5. The net effect will be to obtain, due to Equation (10), and Equation (11), and use $a \equiv\left[a_{0}=1\right] /(1+z)$. As given by Beckwith [7]

$$
q=-\frac{\ddot{a} a}{\dot{a}^{2}} \equiv-1+\frac{2}{1+\tilde{\kappa}^{2}[\rho / m] \cdot(1+z)^{4} \cdot(1+\rho / 2 \lambda)}
$$

Equation (10) assumes $\Lambda=0=K$, and the net effect is to obtain, a substitute for $\mathrm{DE}$, by presenting how gravitons with a small mass done with $\Lambda \neq 0$, even if curvature $K=0$. The 'density' function, $\rho$, assumed is similar to what was done by Alves [11], et al.

\subsection{Consequences of Small Graviton Mass for Reacceleration of the Universe}

Using Equation (12) leads to the prediction given in Figure 1 below:

Now that this is presented, we should consider what the effects of a small graviton mass would be for initialentropy/information counting at the onset of inflation.

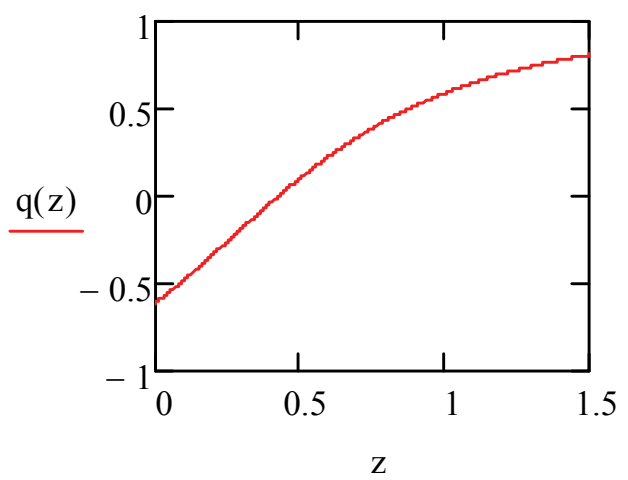

Figure 1. Reacceleration of the universe based on Beckwith [7] (note that $\mathrm{q}<0$ if $\mathrm{z}<.423$ ).

\section{Examination of Weyl Scalar in the Onset of Inflation, to Obtain Entropy Counting Initially?}

Note, from Valev [12]

$$
\begin{aligned}
& \left.m_{\text {graviton }}\right|_{\text {RELATIVISTIC }}<4.4 \times 10^{-22} \mathrm{~h}^{-1} \mathrm{eV} / \mathrm{c}^{2} \\
& \Leftrightarrow \lambda_{\text {graviton }} \equiv \frac{\hbar}{m_{\text {graviton }} \cdot \mathrm{c}}<2.8 \times 10^{-8} \text { meters }
\end{aligned}
$$

This could be an argument with non zero graviton mass what to expect as a way to formulate our $n_{f}$ which would permit, if given a frequency range more precise ways to obtain $n_{f}$, so as to find a better way to use Equation (1) more effectively. To start this, look at setting, with $\hat{a}$ a radiation constant, and using a value given by Sander's [13] with an observationally based Friedman Equation based value of

$$
H_{\text {friedman }} \equiv\left[\left(T_{\text {temp }}\right)^{2} / c\right] \cdot \sqrt{N\left(T_{\text {temp }}\right)} \cdot \sqrt{\frac{4 \pi G \cdot \hat{a}}{3}}
$$

So, then that, if one uses $N\left(T_{\text {temp }}\right) \sim 10^{3}$, as opposed to an upper limit specified by Kolb, et al of, at or after electro weak, $N\left(T_{\text {temp }}\right) \sim 10^{2}$, that then

$$
\begin{aligned}
& h^{+} \sim h^{x} \sim \frac{1}{2 r^{2}} \cdot \frac{H_{\text {friedman }}}{m_{\text {Planck }}} \equiv \\
& \frac{1}{2 r^{2}} \cdot\left[\frac{\left(T_{\text {temp }}\right)^{2} / c}{m_{\text {Planck }}}\right] \cdot \sqrt{N\left(T_{\text {temp }}\right)} \cdot \sqrt{\frac{4 \pi G \cdot \hat{a}}{3}}
\end{aligned}
$$

For the sake of inflationary applications, we will assume that $\sqrt{N\left(T_{\text {temp }}\right)} \cdot \sqrt{\frac{4 \pi G \cdot \hat{a}}{3}}$ has no spatial dependence worth speaking of, which leads to, if $\frac{\left(T_{\text {temp }}\right)^{2} / c}{m_{\text {Planck }}} \propto$ constant of order unity. Also $N\left(T_{\text {temp }}\right) \approx 10^{2}-10^{3}$. Then one has

$$
E_{\text {initial }-f l u x} \sim\left[\frac{3 \cdot N\left(T_{\text {temp }}\right)}{64 \pi \cdot r^{6}}\right] \cdot\left[\tilde{n} \cdot t_{\text {Planck }}\right]^{3} \cdot \Omega_{\text {effective }}
$$

For the sake of argument, Beckwith used $\Omega_{\text {effective }}<4 \pi$, and experimented with values of $r<10^{-16} \mathrm{~m}$, and also, of $\tilde{n} \cdot t_{\text {Planck }} \approx 2 \times 10^{-35} \mathrm{sec}, E_{\text {initial }- \text { flux }} \sim 10^{-20} \mathrm{eV}$. If one uses the $m_{\text {graviton }} \sim 10^{-65}$ grams, then Beckwith obtained $n_{f} \approx 10^{6}$ to $\cdot 10^{7}$. This is assuming a very high initial frequency for the relic particles. We will in the next section comment upon. 


\section{Conclusions. Examining Information Exchange between Different Universes?}

Beckwith [7] has concluded that the only way to give an advantage to higher dimensions as far as cosmology would be to look at if a fifth dimension may present a way of actual information exchange to give the following parameter input from a prior to a present universe, i.e. the fine structure constant, as given by [7]

$$
\tilde{\alpha} \equiv e^{2} / \hbar \cdot c \equiv \frac{e^{2}}{d} \times \frac{\lambda}{h c}
$$

The wave length as may be chosen to do such an information exchange would be part of a graviton as being part of an information counting algorithm as can be put below, namely:

Argue that when taking the log, that the $1 / \mathrm{N}$ term drops out. As used by $\mathrm{Ng}$ [5]

$$
Z_{N} \sim(1 / N !) \cdot\left(V / \lambda^{3}\right)^{N}
$$

This, according to $\mathrm{Ng}$, [5] leads to entropy of the limiting value of, if $S=\left(\log \left[Z_{N}\right]\right)$ will be modified by having the following done, namely after his use of quantum infinite statistics, as commented upon by Beck with [7]

$$
S \approx N \cdot\left(\log \left[V / \lambda^{3}\right]+5 / 2\right) \approx N
$$

Eventually, the author hopes to put on a sound foundation what 'tHooft [14] is doing with respect to t'Hooft [14] deterministic quantum mechanics and equivalence classes embedding quantum particle structures. Furthermore, making a count of gravitons with $S \approx N \sim 10^{7}$ gravitons [7], with Seth Lloyd's [15]

$$
I=S_{\text {total }} / k_{B} \ln 2=[\# \text { operations }]^{3 / 4} \sim 10^{7}
$$

as implying at least one operation per unit graviton, with gravitons being one unit of information, per produced graviton [7]. Note, Smoot [4] gave initial values of the operations as

$$
\text { [\#operations }]_{\text {initially }} \sim 10^{10}
$$

The argument so presented, gives a first order approximation as to how to obtain Equation (21)

\section{References}

[1] M. Maggiore, "Gravitational Waves, Volume 1: Theory and Experiment," Oxford University Press, Oxford, 2008.

[2] R. Durrer and M. Rinaldi, Physical Review, D79: 063507, 2009, http://arxiv.org/abs/0901.0650. doi:10.1103/PhysRevD.79.063507

[3] M. Alcubierre, "Introduction to Numerical Relativity," Oxford University Press, 2008. doi:10.1093/acprof:oso/9780199205677.001.0001

[4] G. Smoot, http://chalonge.obspm.fr/Paris07_Smoot.pdf

[5] Y. Ng, Entropy, Vol. 10, No. 4, 2008, pp. 441-461. doi:10.3390/e10040441

[6] M. Marklund, G. Brodin and P. Shukla, Physica Scripta Vol. T82, 1999, pp. 130-132.

[7] A. Beckwith, http://vixra.org/abs/0912.0012, v 6 (newest version).

[8] R. Maartens, "Brane-World Gravity," http://www.livingrev iews.org//rr-2004-7, 2004; R. Maartens, "Brane World Cosmology," Lecture Notes in Physics, Vol. 653, Springer Verlag, Berlin, 2005, pp. 213-247.

[9] V. Rubakov, "Classical Theory of Gauge Fields," Princeton University Press, Princeton, 2002.

[10] S. Dubovsky, R. Flauger, A. Starobinsky and I. Tkachev, report UTTG-06-09, TCC-23- 09. http://arxiv.org/abs/0907. 1658

[11] E. Alves, O. Miranda and J. de Araujo, arXiv: 0907.5190 (July 2009).

[12] D. Valev, Aerospace Research in Bulgaria, Vol. 22, 2008, pp. 68-82. http://arxiv.org/abs/hep-ph/0507255; http:// arxiv.org/abs/1004.2449.

[13] R. Sanders, "Observational Cosmology," pp. 105-137, in "The Physics of the Early Universe," Lecture Notes in Physics, pp. 653, E. Papantonopoulou, Ed., Springer Verlag, Berlin, 2005.

[14] G. 't Hooft, http://arxiv.org/PS cache/quant-ph/pdf/0212/ 0212095v1.pdf, 2002; G. 't Hooft., In: Th. M. Nieuwenhuizen et al., Eds., Beyond the Quantum, World Press Scientific, http://arxiv.org/PS_cache/quant-ph/pdf/0604/ 0604008v2.pdf, 2006.

[15] S. Lloyd, Physical Review Letters, Vol. 88, 237901, 2002. 